\title{
Validity of the hyperbolic Whitham modulation equations in Sobolev spaces
}

\author{
Thomas J. Bridges ${ }^{1}$, Anna Kostianko ${ }^{1,2}$, and Sergey Zelik ${ }^{1,2}$ \\ 1. Department of Mathematics, University of Surrey, Guildford GU2 7XH, UK1 \\ 2. School of Mathematics and Statistics, Lanzhou University, Lanzhou 730000 P.R. China
}

\begin{abstract}
It is proved that modulation in time and space of periodic wave trains, of the defocussing nonlinear Schrödinger equation, can be approximated by solutions of the Whitham modulation equations, in the hyperbolic case, on a natural time scale. The error estimates are based on existence, uniqueness, and energy arguments, in Sobolev spaces on the real line. An essential part of the proof is the inclusion of higher-order corrections to Whitham theory, and concomitant higher-order energy estimates.
\end{abstract}

\section{Introduction}

We study the validity of slow modulation of space-time periodic solutions of the cubic nonlinear Schrödinger (NLS) equation

$$
\mathrm{i} \partial_{t} \Psi+\partial_{x}^{2} \Psi+\gamma|\Psi|^{2} \Psi=0,
$$

where $\Psi(t, x)$ is complex valued, $\gamma= \pm 1, x \in \mathbb{R}$ and $t \geq 0$. This equation possesses an exact three-parameter family of plane wave solutions

$$
\Psi(t, x)=\Psi_{0}(\omega, k) \mathrm{e}^{\mathrm{i} \theta}, \quad \theta=\omega t+k x+\theta_{0},
$$

where $\Psi_{0}$ is real and positive, $\theta_{0}$ is a constant, and $(\omega, k)$ are the frequency and wave number. Substitution into (1.1) gives the nonlinear dispersion relation: $\gamma \Psi_{0}^{2}=\omega+k^{2}$.

Modulated solutions are obtained by introducing the standard geometric optics ansatz:

$$
\Psi(t, x)=\widetilde{\Psi}(T, X, \varepsilon):=\widetilde{A}(T, X, \varepsilon) e^{\mathrm{i} \varepsilon^{-1}(\omega T+k X+\widetilde{\phi}(T, X, \varepsilon))},
$$

where $\varepsilon \ll 1$ is a small parameter, $T=\varepsilon t, X=\varepsilon x$ and $\widetilde{A}(T, X, \varepsilon)$ and $\widetilde{\phi}(T, X, \varepsilon)$ are the slowly varying amplitude and phase respectively. Substituting (1.3) into (1.1), separation of real and imaginary parts, taking the limit $\varepsilon \rightarrow 0$, and differentiating the real part with respect to $X$, gives the Whitham modulation equations (WMEs) in the form

$$
\partial_{T} A+2(k+u) \partial_{X} A+A \partial_{X} u=0 \quad \text { and } \quad \partial_{T} u+2(k+u) \partial_{X} u-2 \gamma A \partial_{X} A=0 .
$$

\footnotetext{
${ }^{1}$ Email: T.Bridges@surrey.ac.uk, Anna.Kostianko@surrey.ac.uk, S.Zelik@surrey.ac.uk
} 
In these equations

$$
A(T, X)=\widetilde{A}(T, X, 0), \quad \phi(T, X)=\widetilde{\phi}(T, X, 0), \text { and } u(T, X):=\partial_{X} \phi(T, X) .
$$

The main aim of this paper is to prove that solutions of the WMEs (1.4) stay close to the exact solution of (1.1), when commensurately initialized, relative to a metric based on the Sobolev space $H^{s}(\mathbb{R})$ for some positive index $s$, when $\varepsilon$ is sufficiently small.

The overarching motivation for this work is validity of the WMEs in general, starting from an abstract Lagrangian, or abstract Euler-Lagrange equation. This target is currently intractable, and progress to date has been achieved by proving validity of reduction to the WMEs for specific equations. Remarkably, validity proofs for the WMEs are rare and the only proofs known to the authors are validity of the reduction from PDEs of Korteweg-de Vries (KdV) type (e.g. Bronski ET AL. [6, 5] and references therein) and validity of the reduction of the NLS equation to the WMEs (e.g. DüLL \& SCHNEIDER [8]). In the same spirit as validity, the paper BenzOni-GAVAGE ET AL. [1 proves, for Hamiltonian PDEs of $\mathrm{KdV}$ type, the link between hyperbolicity of the WMEs and the spectral stability of periodic traveling waves to sideband perturbations.

In this paper the strategy is to start with a fairly simple PDE, the cubic NLS equation, but give comprehensive results on how solutions of the WMEs (1.4) stay close to the exact solution of (1.1) in Sobolev spaces. Local existence and uniqueness of solutions of both (1.1) and (1.4) is relatively straightforward (the latter given in \$4, the former a consequence of the proof in 5 ). Indeed, with $A=\sqrt{h}$ and $\gamma=-1$ the equations (1.4) are just a variant of the classical shallow water equations (SWEs). However, in our study of (1.4) we introduce higher-order energies which feed into a proof of validity of higher-order Whitham theory in \$5. Subtleties do arise when we start comparing the solutions of (1.4) to solutions of (1.1). The most surprising of which is that it will be essential to include higher-order Whitham theory in the approximation

$$
\widehat{A}(T, X, \varepsilon)=A(T, X)+\varepsilon^{2} A_{1}(T, X)+\cdots+\varepsilon^{2 n} A_{n}(T, X),
$$

for some fixed and finite $n$, with similar expansions for the other variables. These series may not be convergent, but only estimates for the existence of a fixed and finite sum of these terms is required. Indeed $n=1$ is sufficient, but our proof is stated in terms of arbitrary but finite $n$. The study of expansions of the form (1.6) is given in \$5, Other idiosyncrasies are recorded in 93 . Before stating the main result, we review the related literature on analysis of NLS, from the WKB, semi-classical and integrability perspectives, and emphasize the new features that arise when we segue into the specifics of the WMEs validity problem.

There is an extensive literature on the rigorous analysis of approximate solutions of NLS (1.1) from the perspective of WKB theory and semi-classical analysis. The most studied case is for vanishing basic state, where $\omega=k=0$ and $\Psi_{0}=0$, and $s$ can be taken to be zero. This case is referred as the semiclassical limit or supercritical nonlinear optics limit and is usually studied by expanding the functions $\widetilde{A}$ and $\widetilde{\phi}$ in Taylor series in $\varepsilon$ similar to (1.6), the socalled WKB expansions (see e.g. CARLES [7] and references therein). In the defocussing case $\gamma=-1$ the leading terms in these expansions are related to the compressible Euler equation (and the SWEs in the 1D case) and, since the local solvability of these equations in Sobolev 
spaces is well-understood (e.g. MAJDA [14]), the recurrent equations for the WKB expansions can be solved (also locally) in Sobolev spaces and the corresponding error estimates deduced (see [7] for details). In the case $\gamma=+1$, the leading order equations are ill-posed and the WKB expansions are more delicate. In this case the WKB expansions can still be effectively studied by working in spaces of analytic functions (e.g. GERARD [9]).

Since the NLS equation (1.1) is integrable, by the Zakharov-Shabat formalism, there is another body of work that incorporates this structure into the analysis, particularly in the study of the semiclassical approximation of the defocussing NLS equation (e.g. Chapter 5 of Kamchatnov [13], Jin, Levermore, \& McLaughlin [12], Carles [7], Grebert \& T. KAPPELER [10] and references therein). In the proofs in this paper integrability of the NLS equation is not used in any way. The main disadvantage being that our results do not give any information about the fine detail of the approximation error, and the structure of the oscillations that arise in the $\varepsilon \rightarrow 0$ limit. The main advantage is that the methodology extends to non-integrable systems.

Modulation of the general case where the basic state is non-trivial, $\Psi_{0}=\Psi_{0}(\omega, k) \neq 0$, which is the principal case of interest in validity of WMEs, is much less studied from the WKB perspective. Although the WKB analysis can be formally performed exactly as before, some new difficulties arise due to the fact that the amplitude $\Psi_{0}$ is not in $L_{2}(\mathbb{R})$. This difficulty, and its appearance in the validity theory, is addressed in $\$ 3$,

Another, probably more essential difficulty comes from the fact that the underlying wavetrain (1.2) may a priori be linearly unstable (which is an essential feature of the selffocussing case). Unfortunately, the longtime validity in Sobolev spaces can not be achieved if the corresponding wavetrain is spectrally unstable.

The validity problem in this case (that is, $\Psi_{0} \neq 0$ and $\gamma= \pm 1$ ) has been studied by DüLL \& SCHNEIDER [8] in the framework of functions which are analytic in a strip about the real axis (so called Gevrey spaces). In this case, the stability/instability properties of the underlying wavetrain are not as important due to the very fast decay of higher Fourier modes provided by the analyticity, so that analysis works in both the focussing and defocussing cases. Validity is proved in [8], locally in time, in Gevrey spaces.

However, as pointed out in [8], the analysis in Gevrey spaces is not entirely satisfactory from a validity perspective, due to the required constraint on the time interval of existence. Indeed, it is usually expected that the WKB approximation will work at least until blow up of the corresponding smooth solution of the limit equations (= formation of caustics in the terminology of geometric optics), but it is not clear how to obtain this in the framework of analytic functions. Indeed, the standard technique is to work with time-dependent Gevrey spaces of analytic functions in a strip, in the complex plane about the real axis, with shrinking size (linearly in time) where the rate of shrinking is determined by the norms of the initial data. For this reason, the width of this strip for the exact solution typically shrinks to zero fast, no matter how long the lifespan of the limit solution is, see [8]. As a result, the limit solution to the initial NLS equation can only be justified on a very small time interval (in slow time), no matter how big its lifespan actually is.

This latter shortcoming motivates us to study the validity of the Whitham approximations for NLS in Sobolev spaces where this problem can potentially be overcome. Indeed, the 
validity of these approximations in the defocussing case in Sobolev spaces is stated as an open problem in DüLL \& SCHNEIDER [8]. Only the defocussing case is suitable for the framework of Sobolev spaces, so we predominantly restrict attention henceforth to the case $\gamma=-1$.

An outline of the paper is as follows. First, before proceeding with the proof of validity, we look more closely at the derivation and properties of the WMEs (1.4) in \$2, The conventional view of Whitham theory based on an averaged Lagrangian is recorded, and then a rigorous derivation of the averaging and approximation process is given from first principles, which may have independent interest.

The validity proof starts in $\$ 4$ with a proof of the local solvability of the WMEs (1.4) in Sobolev spaces. The key observation here is its equivalence to the SWEs for which the local well-posedness is well understood. We utilize the shallow water energy and its natural analogues in higher order Sobolev spaces in order to get the desired result.

At the next step we verify in $\$ 5$ the solvability of the recurrent equations for the higher order approximations to Whitham theory (1.6). Since these equations are linear at leading order and have the same structure as the linearised SWEs, we can construct these expansions using the same energy technique, already developed in $\$ 4$. It is this construction that enables estimates for the residual terms to be pushed up to order $\varepsilon^{2 n}$ in the theorem below.

In $\$ 6$ we tie all the results together by proving rigorous estimates on the comparison between the exact solution $\widetilde{\Psi}$,

$$
\widetilde{\Psi}(T, X, \varepsilon)=\widehat{\Psi}(T, X, \varepsilon)(1+W(T, X, \varepsilon)),
$$

and the approximate solutions $\widehat{\Psi}$ in (1.3), of the NLS equation (1.1). In (1.7), the complexvalued function $W$ (which measures the deviation of $\widehat{\Psi}$ from $\widetilde{\Psi}$ ) satisfies an exact, but singularly perturbed, version of the NLS equation, and it is derived in $\$ 6$. We prove the smallness of $W$ using a special energy type estimate inspired by the proof of the spectral stability of the underlying wavetrain. The main result of the paper, stated here, is proved in $\$ 6$.

Theorem 1.1. Let $n \geq 1$ in (1.6) and fixed, and suppose the initial data for the NLS equation (1.1) with $\gamma=-1$ has the form

$$
\Psi(0, x)=e^{r_{0}(\varepsilon x)} e^{i\left(k x+\varepsilon^{-1} \phi_{0}(\varepsilon x)\right)}, \quad \varepsilon \ll 1,
$$

for some constants $\omega$ and $k$ satisfying $\omega+k^{2}+1=0$ and some functions $\left(r_{0}, \phi_{0}\right)$ of the slow variable $X=\varepsilon x$ satisfying

$$
\left\|r_{0}\right\|_{H^{3 n+5}(\mathbb{R})}+\left\|\partial_{X} \phi_{0}\right\|_{H^{3 n+5}(\mathbb{R})} \leq C,
$$

where the constant $C$ is independent of $\varepsilon$. Assume also that the associated WMEs (1.4) with the initial data $\left(r_{0}, \phi_{0}\right)$ possess a smooth solution on some time interval $T \leq T_{0}$ which satisfies

$$
\|r(T)\|_{H^{3 n+5}(\mathbb{R})}+\|u(T)\|_{H^{3 n+5}(\mathbb{R})} \leq C, \quad T \leq T_{0},
$$

for some new constant $C$ also independent of $\varepsilon$, where $r(T, X)=\ln A(T, X)$. Then the exact solution $\widetilde{\Psi}(T, X, \varepsilon)$, of the NLS equation with the same initial data, exists on a time 
interval $T \in\left[0, T_{0}\right]$ and remains $\varepsilon^{2 n}$-close to the appropriate $n$th order Whitham modulation approximation $\widehat{\Psi}=\widehat{A} e^{i \varepsilon^{-1} \widehat{\Theta}}$ in the following sense:

$$
\left\|(\widetilde{\Psi}(T, \cdot)-\widehat{\Psi}(T, \cdot)) e^{-i \varepsilon^{-1} \widehat{\Theta}(T, \cdot)}\right\|_{H^{1}(\mathbb{R})} \leq C \varepsilon^{2 n}, \quad T \leq T_{0},
$$

where the constant $C$ is independent of $\varepsilon$.

Remark 1.2. Using the fact that $H^{1} \subset L^{\infty}$, and under the assumptions of the theorem, we can also compare the solutions $\widehat{\Psi}$ and $\widetilde{\Psi}$ without the phase factor $e^{\mathrm{i} \varepsilon^{-1}} \widehat{\Theta}$ :

$$
\|\widetilde{\Psi}(T, \cdot)-\widehat{\Psi}(T, \cdot)\|_{L^{\infty}(\mathbb{R})} \leq C \varepsilon^{2 n}, \quad T \leq T_{0}
$$

However, if we want to compare this distance in higher Sobolev norms, it will be necessary to decrease the order of approximation due to the factor $\varepsilon^{-1}$ in the phase.

Remark 1.3. We also would like to emphasize that the order of approximation in estimate (1.9) in Theorem 1.1 is $\varepsilon^{2 n}$, not $\varepsilon^{2(n+1)}$ as one might expect. For this reason, we can not take $n=0$ in (1.6) and need to retain at least one higher-order term in (1.6). It is not clear whether this restriction is technical, or something more fundamental is behind it.

\section{Variations on Whitham modulation theory}

The modulation ansatz (1.3) is just a geometric optics approximation. What makes it Whitham modulation theory (WMT) is the identification of the first equation in (1.4) with the "conservation of wave action" and the second equation in (1.4) with the "conservation of waves". Also important in Whitham theory is that an averaged Lagrangian is the organising centre. It is this triple, and the generation of conservation of wave action via Noether's Theorem from the averaged Lagrangian, that gives WMT its universality. Background on WMT can be found in Whitham [16], Kamchatnov [13], Bridges [2], and references therein.

In this section, we discuss the steps leading up to the WMEs from first principles starting with an abstract Lagrangian for a general conservative PDE. Let $L=L\left(\mathbf{v}_{t}, \mathbf{v}_{x}, \mathbf{v}\right)$ be a given smooth function of the vector valued function $\mathbf{v}(t, x)$ for $t, x \in \mathbb{R}$, and suppose that the variational principle

$$
\delta \int_{t_{1}}^{t_{2}} \int_{x_{1}}^{x_{2}} L\left(\mathbf{v}_{t}, \mathbf{v}_{x}, \mathbf{v}\right) \mathrm{d} x \mathrm{~d} t=0
$$

with fixed endpoints on the variations $\delta \mathbf{v}$ generates the governing equations. The corresponding Euler-Lagrange equation reads

$$
\partial_{t}\left(L_{\mathbf{v}_{t}}\left(\mathbf{v}_{t}, \mathbf{v}_{x}, \mathbf{v}\right)\right)+\partial_{x}\left(L_{\mathbf{v}_{x}}\left(\mathbf{v}_{t}, \mathbf{v}_{x}, \mathbf{v}\right)\right)=L_{\mathbf{v}}\left(\mathbf{v}_{t}, \mathbf{v}_{x}, \mathbf{v}\right)
$$

Now assume that this equation possesses a three parameter family of single-phase space-time periodic solutions

$$
\mathbf{v}(t, x):=\mathbf{v}_{0}(\theta, \omega, k), \quad \theta=\omega t+k x+\theta_{0}, \omega, k, \theta_{0} \in \mathbb{R},
$$

where $\mathbf{v}_{0}(s, \omega, k)$ is a smooth function, which is $2 \pi$-periodic with respect to $s$ for all $\omega$ and $k$. Inserting this solution into the Euler-Lagrange equation, gives us a differential equation for $\mathbf{v}_{0}(s, \omega, k)$ 


$$
\begin{aligned}
\left(L_{\mathbf{v}_{t}, \mathbf{v}_{t}}^{\prime \prime}\left(\partial_{s} \mathbf{v}_{0} \omega, \partial_{s} \mathbf{v}_{0} k, \mathbf{v}_{0}\right) \omega^{2}+2 L_{\mathbf{v}_{t}, \mathbf{v}_{x}}^{\prime \prime}(\cdots) \omega k+L_{\mathbf{v}_{x}, \mathbf{v}_{x}}^{\prime \prime}(\cdots) k^{2}\right) \partial_{s}^{2} \mathbf{v}_{0}+ \\
+\left(L_{\mathbf{v}_{t}, \mathbf{v}}^{\prime \prime}(\cdots) \omega+L_{\mathbf{v}_{x}, \mathbf{v}}^{\prime \prime}(\cdots) k\right) \partial_{s} \mathbf{v}_{0}=L_{\mathbf{v}}^{\prime}(\cdots) .
\end{aligned}
$$

Motivated by geometric optics theory, we seek for a modulated solution of (2.1) in the form

$$
\mathbf{v}(t, x)=\mathbf{v}_{0}\left(\varepsilon^{-1} \Theta(T, X, \varepsilon), \Omega(T, X, \varepsilon), Q(T, X, \varepsilon)\right)+\varepsilon W(T, X, \varepsilon)
$$

where $\varepsilon \ll 1, T=\varepsilon t, X=\varepsilon x$ are slow variables. The unknown functions $\Theta(T, X, \varepsilon)$, $\Omega(T, X, \varepsilon)$ and $Q(T, X, \varepsilon)$ are slowly-varying functions which are responsible for evolution along the wavetrain manifold, and the function $W(T, X, \varepsilon)$ measures the evolution in transversal directions. Usually some kind of point-wise orthogonality conditions for $\mathbf{v}_{0}$ and $W$ are posed in order to determine $W$ in a unique way, however, we have $W \equiv 0$ in our particular case of NLS, so we prefer not to specify any such conditions here.

In order to (formally) get the approximate equations for quantities $\Theta, \Omega$ and $Q$, we substitute the ansatz (2.4) into the Lagrangian while dropping the terms of order $\varepsilon$ and higher. This gives us the truncated Lagrangian

$$
L_{0}\left(s, \Theta_{X}, \Theta_{T}, \Omega, Q\right):=L\left(\partial_{s} \mathbf{v}_{0}(s, \Omega, Q) \Theta_{T}, \partial_{s} \mathbf{v}_{0}(s, \Omega, Q) \Theta_{X}, \mathbf{v}_{0}(s, \Omega, Q)\right), \quad s:=\varepsilon^{-1} \Theta
$$

However, this Lagrangian still contains rapidly oscillating terms related with $\varepsilon^{-1} \Theta$, so we need to (again formally) introduce averaging to get the reduced Lagrangian

$$
\mathcal{L}\left(\Theta_{T}, \Theta_{X}, \Omega, Q\right)=\left\langle L_{0}\left(s, \Theta_{T}, \Theta_{X}, \Omega, Q\right)\right\rangle_{s}
$$

where $\langle f\rangle_{s}=\frac{1}{2 \pi} \int_{0}^{2 \pi} f(s) d s$. Taking variational derivatives with respect to $\Omega$ and $Q$, we end up with two equations

$$
\left\{\begin{array}{l}
\left\langle L_{\mathbf{v}_{t}}^{\prime} \partial_{s \omega}^{2} \mathbf{v}_{0}\right\rangle_{s} \Theta_{T}+\left\langle L_{\mathbf{v}_{x}}^{\prime} \partial_{s \omega}^{2} \mathbf{v}_{0}\right\rangle_{s} \Theta_{X}=-\left\langle L_{\mathbf{v}}^{\prime} \partial_{\omega} \mathbf{v}_{0}\right\rangle_{s} \\
\left\langle L_{\mathbf{v}_{t}}^{\prime} \partial_{s k}^{2} \mathbf{v}_{0}\right\rangle_{s} \Theta_{T}+\left\langle L_{\mathbf{v}_{x}}^{\prime} \partial_{s k}^{2} \mathbf{v}_{0}\right\rangle_{s} \Theta_{X}=-\left\langle L_{\mathbf{v}}^{\prime} \partial_{k} \mathbf{v}_{0}\right\rangle_{s}
\end{array}\right.
$$

which are satisfied point-wise for all $T, X \in \mathbb{R}$. This is the linear system with respect to the variables $\Theta_{T}$ and $\Theta_{X}$. We claim that

$$
\Theta_{T}=\Omega, \quad \Theta_{X}=Q
$$

solves this system. A novelty here is that conservation of waves is deduced from the averaged Lagrangian rather than assuming it a priori.

The assertion (2.8) can be verified by integrating by parts the integrals in the left-hand side of (2.7) (moving the $s$-derivative from the function $\mathbf{v}_{0}$ to $L_{\mathbf{v}_{t}}^{\prime}$ and $L_{\mathbf{v}_{x}}^{\prime}$ and using the identity (2.3) $)$. In general, the determinant of the system (2.7) does not vanish identically, so the solution (2.7) is unique. However, it may be not so in degenerate cases like our NLS example which will be considered in the subsection below.

Inserting (2.8) into the reduced Lagrangian, we finally arrive at the standard WME averaged Lagrangian

$$
\mathcal{L}_{\mathrm{WME}}(\Omega, Q):=\left\langle L_{0}(s, \Omega, Q, \Omega, Q)\right\rangle_{s},
$$


which should be considered under the extra constraint

$$
\Omega_{X}=Q_{T} .
$$

The associated Euler-Lagrange equations finally give us the desired Whitham modulation equations (WMEs) in a standard form

$$
\partial_{T} \mathscr{A}(\Omega, Q)+\partial_{X} \mathscr{B}(\Omega, Q)=0,
$$

with $\mathscr{A}:=\partial_{\Omega} \mathscr{L}_{\mathrm{WME}}$ and $\mathscr{B}=\partial_{Q} \mathscr{L}_{\mathrm{WME}}$. The equation (2.11) is called "conservation of wave action" in [16]. Of course, this equation should be considered together with (2.10) which gives a closed system of two quasi-linear first order equations for determining the wave frequency $\Omega$ and wave number $Q$. The wave phase $\Theta$ is determined after that from the relation

$$
d \Theta=\Omega d T+Q d X
$$

and the exactness of this differential form is guaranteed by (2.10). Using (2.8) these equations can be also rewritten in terms of a single second order quasi-linear PDE for the phase $\Theta$ :

$$
\partial_{T} \mathscr{A}\left(\Theta_{T}, \Theta_{X}\right)+\partial_{X} \mathscr{B}\left(\Theta_{T}, \Theta_{X}\right)=0 .
$$

Remark 2.1. There is an effective machinery which allows us to compute the WME Lagrangian $\mathcal{L}_{\mathrm{WME}}(\omega, k)$, namely, we may just put the family of solutions (2.2) where $\omega$ and $k$ are constants into the initial Lagrangian $L$ and compute the result as a function of $\theta, \omega$ and $k$. Performing thereafter averaging with respect to the variable $\theta$, we end up exactly with the WME lagrangian $\mathcal{L}_{\mathrm{WME}}(\omega, k)$, see (2.5), (2.6) and (2.9). These steps are essentially how the scheme is usually presented in the literature, see e.g. [16, 8, 2]. However, in the conventional approach the extra constraint (2.10) is just postulated. For this reason, we have given a bit more detailed derivation here of the WMEs which includes the emergence of this constraint as well. Moreover, the precise form (2.4) that we are seeking for the modulated solution is also important for the forthcoming justification of the above formal procedures. Finally, we have presented here the result for a general Lagrangian $L\left(\mathbf{v}_{t}, \mathbf{v}_{x}, \mathbf{v}\right)$ since the particular case of the NLS Lagrangian is "too degenerate" to see the key features of the theory.

\subsection{WMT for the cubic NLS equation}

Now restrict attention to the cubic NLS equation, which is generated by the Lagrangian

$$
L=\frac{1}{2} \mathrm{i}\left(\bar{\Psi} \partial_{t} \Psi-\Psi \bar{\partial}_{t} \Psi\right)-\left|\partial_{x} \Psi\right|^{2}+\frac{1}{2} \gamma|\Psi|^{4} .
$$

The Euler-Lagrange equation, obtained by taking variations of the integral of $L$ with fixed endpoint conditions, is the cubic NLS (1.1).

The family of space-time periodic solutions (2.2) is given explicitly by

$$
\Psi_{0}(\theta, \omega, k):=\Psi_{0}(\omega, k) \mathrm{e}^{\mathrm{i} \theta}, \quad \text { with } \gamma \Psi_{0}^{2}(\omega, k)=\omega+k^{2} .
$$

Express the amplitude as $\Psi_{0}(\omega, k)=A(\omega, k)$, as when modulated it will be the amplitude in the WMEs. Then the ansatz (2.4) for the modulated solution reads

$$
\Psi(t, x)=A(\Omega(T, X), Q(T, X)) \mathrm{e}^{\mathrm{i} \frac{1}{\varepsilon} \Theta(T, X)} .
$$


In this case we do not have any transversal directions to the wavetrain manifold, so $W \equiv 0$. This observation does not affect the leading order approximate equations (which do not contain $W$ in any case), but it is crucial when higher order approximations are considered, see next section.

The truncated Lagrangian $L_{0}$ now reads

$$
L_{0}\left(s, \Theta_{T}, \Theta_{X}, \Omega, Q\right)=-\frac{1}{2} \gamma\left(A^{4}\left(\Theta_{T}, \Theta_{X}\right)-\left(A^{2}(\Omega, Q)-A^{2}\left(\Theta_{T}, \Theta_{X}\right)\right)^{2}\right)
$$

and we see two important simplifications:

1) In contrast to the general case, the truncated Lagrangian is independent of $s$, so there is nothing to average here. This also makes the validity theory essentially simpler.

2) The Lagrangian $L_{0}=\mathcal{L}$ does not depend on $\Omega$ and $Q$ separately, but only on their combination $A^{2}:=\gamma^{-1}\left(\Omega+Q^{2}\right)$. For this reason, the functions $\Omega$ and $Q$ are not uniquely defined, but only their combination $A^{2}$ is. In particular, in this case, the determinant of system (2.7) vanishes identically, so from these equations we get only the relation

$$
A^{2}(\Omega, Q)=A^{2}\left(\Theta_{T}, \Theta_{X}\right) \Longleftrightarrow \Omega+Q^{2}=\Theta_{T}+\Theta_{X}^{2},
$$

but for consistency with the general case, we may define $\Omega=\Theta_{T}$ and $Q=\Theta_{X}$.

The WME Lagrangian now reads

$$
\mathcal{L}_{\mathrm{WME}}\left(\Theta_{T}, \Theta_{X}\right)=-\frac{1}{2} \gamma\left(\Theta_{T}+\Theta_{X}^{2}\right)^{2}
$$

and the corresponding WME has the following form

$$
\partial_{T}\left(\Theta_{T}+\Theta_{X}^{2}\right)+2 \partial_{X}\left(\Theta_{X}\left(\Theta_{T}+\Theta_{X}^{2}\right)\right)=0 .
$$

Remarkably, this equation is independent of $\gamma \neq 0$. Moreover, it is not difficult to see that it is hyperbolic if $\Theta_{T}+\Theta_{X}^{2}<0$, and elliptic if $\Theta_{T}+\Theta_{X}^{2}>0$. It is also straightforward to show that the sign of $\Theta_{T}+\Theta_{X}^{2}$ is preserved under the time evolution (at least until the solution is smooth). Keeping in mind that $\Theta_{T}+\Theta_{X}^{2}=\gamma A^{2}$ and the amplitude $A$ is real, we conclude that $\gamma>0$ corresponds to the elliptic case and in the case $\gamma<0$ the equation is hyperbolic.

It is natural to rewrite equation (2.18) with respect to new variables

$$
h(T, X):=A^{2}(T, X)=\gamma^{-1}\left(\Theta_{T}+\Theta_{X}^{2}\right)
$$

and $u(T, X):=\partial_{X} \Theta(T, X)$. This gives

$$
\partial_{T} u+\partial_{X}\left(u^{2}-\gamma h\right)=0 \text { and } \partial_{T} h+2 \partial_{X}(u h)=0 .
$$

In the hyperbolic case $\gamma<0$ this system is the classical SWEs (up to scaling $2 u \rightarrow u$ ), and we will utilize the connection between the hyperbolic WMEs and the classical SWEs in our proof of validity, in order to get the local solvability of WMEs in Sobolev spaces. In order to handle the degeneracy of the SWEs energy at $h=0$, it is also useful to write the amplitude $A$ in the form $A=e^{r}$ which leads to the following equations for $(r, u)$,

$$
\begin{aligned}
& \partial_{T} r+2 u \partial_{X} r+\partial_{X} u=0 \\
& \partial_{T} u+2 u \partial_{X} u-2 \gamma \mathrm{e}^{2 r} \partial_{X} r=0 .
\end{aligned}
$$




\section{Validity theory: setup and roadmap}

There are several dimensions to the approximation theory. The backbone is the usual three steps in validity theory: an existence theory for the original equation, an existence theory for the reduced equation, and the evolution of a measure of the distance between the two (e.g. Part IV of Uecker \& Schneider [15]). In addition, idiosyncrasies arise that are particular to the context of the NLS to WMEs reduction in Sobolev spaces.

The first key question is the choice of function space. In validity theory, our main interest is the case where the basic state (1.2) is non-trivial, $\Psi_{0} \neq 0$, and this solution is not square integrable on the real line and so, in contrast to WKB theory [7], we cannot assume that the amplitude $A(T) \in L^{2}(\mathbb{R})$. It would be natural to consider $A(T) \in L^{\infty}(\mathbb{R})$ or $A(T)$ belonging to some uniformly local Sobolev space, but this is problematic since the NLS equation is not well-posed in such spaces. As a compromise, we will assume that

$$
\lim _{X \rightarrow \pm \infty} A(T, X)=\Psi_{0}, \quad \gamma \Psi_{0}^{2}=\omega+k^{2},
$$

so the amplitude of the modulated solution stabilizes as $X \rightarrow \pm \infty$ to the amplitude of the basic wavetrain. The same assumption will be posed also for the amplitudes $\widetilde{A}(T, X, \varepsilon)$ of exact solutions of the NLS equation as well as their $n$th order approximations $\widehat{A}(T, X, \varepsilon)$.

In contrast to [8] where the problem has been considered in spaces of analytic functions, we cannot treat the elliptic case $\gamma>0$ in Sobolev spaces (elliptic equations are usually ill-posed in such spaces), so we have to assume that $\gamma<0$. Taking into the account that $\omega+k^{2} \neq 0$, we may scale the variables $t, x$ and $\Psi$ in NLS and assume without loss of generality that

$$
\gamma=-1, \quad \omega+k^{2}+1=0, \quad \Psi_{0}=1 .
$$

A valuable simplification that arises in the case $\Psi_{0} \neq 0$ is that we may naturally separate the amplitude $A$ from the singularity at $A=0$ by assuming that

$$
\ln \frac{A(T, \cdot)}{\Psi_{0}(\omega, k)} \in H^{s}(\mathbb{R}), \quad \text { for some index } s>0 .
$$

We will prove below that if this assumption is satisfied for $T=0$ it will be preserved at least for small positive time $T \leq T_{0}$, and the same is true when we replace $A$ by $\widetilde{A}$ and $\widehat{A}$ in (3.2). This assumption is equivalent to

$$
A(T, \cdot)-\Psi_{0} \in H^{s}(\mathbb{R}) .
$$

The fact that the NLS equation is ill posed in $L^{\infty}(\mathbb{R})$, as well as in uniformly local Sobolev spaces, makes the assumption (3.2) appear to be unavoidable here.

Another overarching assumption is

$$
u(T, \cdot):=\partial_{X} \phi(T, \cdot) \in H^{s}(\mathbb{R}), \quad T \geq 0,
$$

for sufficiently large positive index $s$, with similar assumptions for exact solutions $(\widetilde{A}, \widetilde{u})$ and their $n$th order Whitham approximations $(\widehat{A}, \widehat{u})$. 
We now discuss assumption (3.3) and the analysis of the phase $\Theta$. The equations for the amplitude and phase in the geometric optics ansatz (1.3), with

$$
\widetilde{A}(T, X, \varepsilon)=e^{\widetilde{r}(T, X, \varepsilon)}, \quad \widetilde{\Theta}(T, X, \varepsilon)=\omega T+k X+\widetilde{\phi}(T, X, \varepsilon)
$$

are

$$
\begin{aligned}
\partial_{T} \widetilde{\phi}+\left(k+\partial_{X} \widetilde{\phi}\right)^{2}-\gamma \mathrm{e}^{2 \widetilde{r}}+\omega-\varepsilon^{2}\left(\partial_{X}^{2} \widetilde{r}+\left(\partial_{X} \widetilde{r}\right)^{2}\right) & =0 \\
\partial_{T} \widetilde{r}+2\left(k+\partial_{X} \widetilde{\phi}\right) \partial_{X} \widetilde{r}+\partial_{X}^{2} \widetilde{\phi} & =0,
\end{aligned}
$$

From the second equation of (3.5) (and the fact that $\widetilde{r}, \partial_{T} \widetilde{r} \in H^{s}$ ), we expect that $\partial_{X}^{2} \widetilde{\phi}$ should be square integrable and $\partial_{X} \widetilde{\phi}$ should be bounded, then differentiating the first equation with respect to $X$, we get that $\partial_{T X}^{2} \widetilde{\phi}$ is square integrable, which together with the comparison with the basic wave train (2.15) gives the natural assumption:

$$
\partial_{T} \widetilde{\phi}, \partial_{X} \widetilde{\phi} \in H^{s}(\mathbb{R})
$$

Note that we do not assume that $\widetilde{\phi}(T) \in H^{s}(\mathbb{R})$, only $\partial_{X} \widetilde{\phi} \in H^{s}(\mathbb{R})$. Actually, in general we do not have even that $\tilde{\phi}(T) \in L^{\infty}(\mathbb{R})$ and it may grow as $X \rightarrow \infty$ slightly slower than $\sqrt{|X|}$. With a slight abuse of notation, we denote

$$
\widetilde{u}(T, X, \varepsilon):=\partial_{X} \widetilde{\phi}(T, X, \varepsilon) .
$$

We do not claim that assumptions (3.2) and (3.6) are the most general for verifying the validity in Sobolev spaces (for example, the slightly more general assumptions, that $\widetilde{u} \in L^{\infty}$ and $\partial_{X} \widetilde{u} \in L^{2}$, also look acceptable, but are more difficult to implement since infinite energy solutions of the shallow water equations should then be considered), they are just convenient and look natural to us. So, from now on we assume that these conditions are always satisfied. In particular, they are satisfied for the initial data at $T=0$ :

$$
\widetilde{r}(0)=r(0):=\ln \widetilde{A}(0) \in H^{s}(\mathbb{R}), \quad \widetilde{u}(0)=u(0):=\partial_{X} \widetilde{\phi}(0) \in H^{s}(\mathbb{R})
$$

for some sufficiently large $s$.

With this setup, our strategy will be as follows. We fix some initial data $(r(0), \phi(0))$ satisfying (3.6) and (3.7) for sufficiently large $s$ and consider the corresponding solution $(r(T), u(T))$ satisfying the WMEs (SWEs) in (2.20), on some interval $T \leq T_{0}$. The local existence and uniqueness of such a solution is proved in $\$ 4$ although we do not assume that $T_{0}$ is small.

Then, formally Taylor expanding the geometric optics representation in $\varepsilon$, in terms of the variables $(\widehat{r}, \widehat{\phi})$, we get the recurrent linear equations for the corresponding Taylor coefficients. In $\$ 5$ it is proved that the obtained recurrent equations are uniquely solvable on the same time interval $T \in\left[0, T_{0}\right]$, so these expansions are well-defined. Truncating Taylor series at the $\varepsilon^{2 n}$-term and denoting the obtained functions by $\widehat{A}(T, X, \varepsilon)$ and $\widehat{\phi}(T, X, \varepsilon)$ respectively, we represent the approximate modulated solution (1.3) of the NLS in the form (3.4). The amplitude and phase of this approximate solution (for $n$ fixed and finite) will satisfy the equations

$$
\begin{aligned}
\partial_{T} \widehat{\phi}+\left(k+\partial_{X} \widehat{\phi}\right)^{2}-\gamma \widehat{A}^{2}+\omega-\varepsilon^{2} \widehat{A}^{-1} \partial_{X}^{2} \widehat{A} & =\operatorname{Res}_{\phi} \\
\partial_{T} \widehat{A}+2\left(k+\partial_{X} \widehat{\phi}\right) \partial_{X} \widehat{A}+\widehat{A} \partial_{X}^{2} \widehat{\phi} & =\operatorname{Res}_{A}
\end{aligned}
$$


with the residual terms $\operatorname{Res}_{\phi}$ and $\operatorname{Res}_{A}$ satisfying

$$
\left\|\operatorname{Res}_{A}(T, \cdot)\right\|_{H^{s^{\prime}}(\mathbb{R})}+\left\|\operatorname{Res}_{\phi}(T, \cdot)\right\|_{H^{s^{\prime}(\mathbb{R})}} \leq C \varepsilon^{2(n+1)}, \quad T \leq T_{0},
$$

for some $s^{\prime}=s^{\prime}(n)<s$ along with

$$
\widehat{r}(T, X, 0)=r(T, X) \text { and } \widehat{\phi}(T, X, 0)=\phi(T, X),
$$

see $\$ 5$ for the details. The results of $\$ 5$ then feed into $\$ 6$, where estimates on the residual between $\widehat{\Psi}$ and the exact solution $\widetilde{\Psi}$ are proved, which combine to complete the proof of Theorem 1.1.

\section{Existence theory for the WMEs in Sobolev spaces}

In this section, we treat the basic WMEs with $\gamma=-1$ from the perspective of the shallow water equations, or more generally as a quasilinear hyperbolic system. The starting point is

$$
\begin{aligned}
& \partial_{T} r=-\partial_{X} u-2(u+k) \partial_{X} r,\left.\quad r\right|_{T=0}=r_{0} \\
& \partial_{T} u=-\partial_{X}(u+k)^{2}-\partial_{X}\left(e^{2 r}\right),\left.\quad u\right|_{T=0}=u_{0} .
\end{aligned}
$$

The arguments that we use are standard in the literature on quasilinear hyperbolic systems (see e.g., [14]), so we will just sketch the proof.

The system (4.1) has an exact energy conservation law with energy

$$
E(r, u)=\left(e^{2 r} u^{2}+\frac{1}{2}\left(e^{2 r}-1\right)^{2}, 1\right)_{L^{2}} \quad \text { satisfying } \quad \frac{d}{d T} E(r, u)=0 .
$$

We start with the analysis of the linearised non-homogeneous problem associated with (4.1)

$$
\left\{\begin{array}{l}
\partial_{T} R=-\partial_{X} U-2 U \partial_{X} r-2(u+k) \partial_{X} R+H_{r}(T),\left.\quad R\right|_{T=0}=R_{0} \\
\partial_{T} U=-2 \partial_{X}((u+k) U)-2 \partial_{X}\left(e^{2 r} R\right)+H_{u}(T),\left.\quad U\right|_{T=0}=U_{0}
\end{array}\right.
$$

where $u(T)$ and $r(T)$ are given smooth functions satisfying

$$
\|r(T)\|_{H^{s+2}}+\|u(T)\|_{H^{s+2}} \leq C, \quad T \leq T_{0} .
$$

We need the following result for this inhomogeneous system. It will also be used later for verifying the existence of local solutions for the non-linear system and in the next section for constructing the higher-order approximate solutions.

Proposition 4.1. Suppose that the smooth functions $r(T)$ and $u(T)$ satisfy (4.4). Then, for every $R_{0}, U_{0} \in H^{s}(\mathbb{R})$ and all external forces $H_{r}, H_{u} \in L^{\infty}\left(0, T_{0} ; H^{s}(\mathbb{R})\right)$, the linear system (4.3) possesses a unique solution $(R, U) \in C\left(0, T_{0} ; H^{s}(\mathbb{R})\right)$ and the following estimate holds:

$$
\begin{aligned}
\|R(T)\|_{H^{s}}^{2}+\|U(T)\|_{H^{s}}^{2} \leq C e^{K T}\left(\|R(0)\|_{H^{s}}^{2}+\|U(0)\|_{H^{s}}^{2}\right)+ & \\
& \quad+C \int_{0}^{T} e^{K(T-s)}\left(\left\|H_{r}(s)\right\|_{H^{s}}^{2}+\left\|H_{u}(s)\right\|_{H^{s}}^{2}\right) d s,
\end{aligned}
$$

where the constants $C$ and $K$ depend only on Sobolev norms of $r$ and $u$. 
Proof. Introduce the energy density and flux

$$
\begin{aligned}
& \widehat{E}_{\text {lin }}(U, R)=\mathrm{e}^{2 r} U^{2}+2 e^{4 r} R^{2} \\
& \widehat{F}_{\text {lin }}(U, R)=2(u+k) \mathrm{e}^{2 r} U^{2}+4(u+k) \mathrm{e}^{4 r} R^{2}+4 \mathrm{e}^{4 r} U R .
\end{aligned}
$$

Then a straightforward calculation, using (4.3), gives

$$
\partial_{T} \widehat{E}_{\text {lin }}+\partial_{X} \widehat{F}_{\text {lin }}=-4 u_{X} \mathrm{e}^{2 r} U^{2}-4 u_{X} \mathrm{e}^{4 r} R^{2}+2 \mathrm{e}^{2 r} U H_{u}+4 \mathrm{e}^{4 r} R H_{r} .
$$

Integrate over $\mathbb{R}$, and use vanishing of the flux $\widehat{F}_{\text {lin }}$ at infinity,

$$
\partial_{T} E_{\text {lin }}=-\left(4 u_{X} \mathrm{e}^{2 r}, U^{2}\right)_{L^{2}}-\left(4 u_{X} \mathrm{e}^{4 r}, R^{2}\right)_{L^{2}}+\left(2 \mathrm{e}^{2 r} U, H_{u}\right)_{L^{2}}+\left(4 \mathrm{e}^{4 r} R, H_{r}\right)_{L^{2}},
$$

where

$$
E_{\operatorname{lin}}=\left(\widehat{E}_{\operatorname{lin}}, 1\right)_{L^{2}} .
$$

Now use the embedding of $H^{1}(\mathbb{R})$ in the space of continuous functions and the CauchySchwarz inequality to arrive at

$$
\frac{d}{d T} E_{\operatorname{lin}}(T) \leq K E_{\operatorname{lin}}(T)+C\left(\left\|H_{u}(T)\right\|_{L^{2}}^{2}+\left\|H_{r}(T)\right\|_{L^{2}}^{2}\right),
$$

where the constants $C$ and $K$ depend only on the norms of second derivatives of $u$ and $r$. The Gronwall inequality now gives the desired estimate (4.5) for $s=0$. For other values of $s$ it can be proved analogously by differentiating (4.3) in space sufficiently many times.

Let us now discuss the case $s=1$ in more detail. Differentiating equations (4.3) in space, we see that the functions $\partial_{X} U$ and $\partial_{X} R$ satisfy the analogue of (4.3) with new right-hand sides

$$
H_{r}^{1}=\partial_{X} H_{r}-2 U \partial_{X}^{2} r+2 \partial_{x} u \partial_{x} R, \quad H_{u}^{1}:=\partial_{X} H_{u}-2 \partial_{X}\left(\partial_{X} u U\right)-4 \partial_{X}\left(\partial_{X} r e^{2 r} R\right) .
$$

Applying the estimate (4.5) with $s=0$ to the equations for $\partial_{X} U$ and $\partial_{X} R$, we arrive at

$$
\begin{aligned}
\left\|\partial_{X} R(T)\right\|_{L^{2}}^{2}+\left\|\partial_{X} U(T)\right\|_{L^{2}}^{2} \leq C e^{K T} & \left(\left\|\partial_{X} R(0)\right\|_{L^{2}}^{2}+\left\|\partial_{X} U(0)\right\|_{L^{2}}^{2}\right)+ \\
& +C \int_{0}^{T} e^{K(T-s)}\left(\left\|H_{r}^{1}(s)\right\|_{L^{2}}^{2}+\left\|H_{u}^{1}(s)\right\|_{L^{2}}^{2}\right) d s .
\end{aligned}
$$

Estimating the $L^{2}$-norms of $H_{r}^{1}$ and $H_{u}^{1}$ using (4.4) with $s=1$ (which gives in particular that $\partial_{X}^{2} u$ and $\partial_{X}^{2} r$ are bounded in $L^{\infty}$ ) and inserting the result into (4.8), we get

$$
\begin{aligned}
& \|R(T)\|_{H^{1}}^{2}+\|U(T)\|_{H^{1}}^{2} \leq C e^{K T}\left(\|R(0)\|_{H^{1}}^{2}+\|U(0)\|_{H^{1}}^{2}\right)+ \\
& +C \int_{0}^{T} e^{K(T-s)}\left(\left\|H_{r}(s)\right\|_{H^{1}}^{2}+\left\|H_{u}(s)\right\|_{H^{1}}^{2}\right) d s+ \\
& \quad+C \int_{0}^{T} e^{K(T-s)}\left(\|U(s)\|_{H^{1}}^{2}+\|R(s)\|_{H^{1}}^{2}\right) d s,
\end{aligned}
$$

where we have also used the estimate (4.5) with $s=0$. Gronwall's inequality applied to (4.9) gives the desired estimate for $s=1$. The case $s>1$ can be done analogously. Thus, the proposition is proved. 
We now turn to the non-linear case and state the main result of this section.

Theorem 4.2. Let $s \geq 2$ and let $u_{0}, r_{0} \in H^{s}(\mathbb{R})$. Then there exists $T_{0}>0$ and $C$, depending only on the $H^{s}$-norms of the initial data, and a unique local solution $(r(T), u(T))$ of problem (4.1) on the time interval $T \in\left[0, T_{0}\right]$ satisfying the estimate

$$
\|r(T)\|_{H^{s}(\mathbb{R})}+\|u(T)\|_{H^{s}(\mathbb{R})} \leq C, \quad \forall T \leq T_{0}
$$

Proof. We want to use the energy conservation law (4.2) to control the $L^{2}$-norm of the solution, namely, utilize the following obvious estimates

$$
e^{-4\|r\|_{L^{\infty}}}\left(\|r\|_{L^{2}}^{2}+\|u\|_{L^{2}}^{2}\right) \leq E(r, u) \leq C e^{4\|r\|_{L^{\infty}}}\left(\|r\|_{L^{2}}^{2}+\|u\|_{L^{2}}^{2}\right) .
$$

However, at the level of $s=0$ the $L^{\infty}$-norm is not under control, and so the energy identity (4.2) is not enough to control these $L^{2}$-norms. So, we need higher energy estimates which we will get by differentiating equations (4.1) in $X$ and using the linearized energies (4.6) $E_{\text {lin }}(R, U)$ for quantities $R=\partial_{X}^{s} r$ and $U=\partial_{X}^{s} u, s=1,2, \cdots$, which satisfy the same analogue of estimate (4.11). As we will see below, $s=1$ is also not enough to close the estimates, so we prove for simplicity the result for $s=2$ only (the first value of $s$ where the estimate can be closed in an elementary way). The case $s>2$ is analogous.

Firstly, to control the $L^{\infty}$-norm of $r$ we use the obvious estimate

$$
\begin{aligned}
\|\| r(T)\left\|_{L^{\infty}}-\right\| r_{0} \|_{L^{\infty}} \mid \leq & T_{0}\left\|r_{T}\right\|_{L^{\infty}} \leq \\
& \leq T_{0}\left(\left\|u_{X}\right\|_{L^{\infty}}+2\|u\|_{L^{\infty}}\left\|r_{X}\right\|_{L^{\infty}}\right) \leq C T_{0}\left(1+\|(r, u)\|_{H^{2}}^{2}\right),
\end{aligned}
$$

for $T \leq T_{0}$. Thus, if we assume that

$$
T_{0} \leq \frac{1}{C\left(1+\|(r(T), u(T))\|_{H^{2}}^{2}\right)}, \quad \text { and } \quad T \leq T_{0}
$$

we will have

$$
\left|\|r(T)\|_{L^{\infty}}-\left\|r_{0}\right\|_{L^{\infty}}\right| \leq 1, \quad \forall T \leq T_{0} .
$$

In turn, this will allow us to replace $\|r(T)\|_{L^{\infty}}$ by $\left\|r_{0}\right\|_{L^{\infty}}$ in equalities like (4.11) and this norm is under control.

Secondly, differentiate equations (4.1) twice in $X$ and let $R:=\partial_{X}^{2} r$ and $U:=\partial_{X}^{2} u$. These functions satisfy linear equations

$$
\begin{aligned}
& R_{T}=-2(u+k) R_{X}-U_{X}-4 u_{X} R-2 r_{X} U \\
& U_{T}=-2(u+k) U_{X}-2 \mathrm{e}^{2 r} R_{X}-6 u_{X} U-12 r_{X} \mathrm{e}^{2 r} R-8 r_{X}^{3} \mathrm{e}^{2 r} .
\end{aligned}
$$

Crucial for us is that the dependence on the second derivatives is linear. Comparison with the equations (4.3) shows that $H_{r}$ and $H_{u}$ are

$$
H_{r}=-4 u_{X} R \text { and } H_{u}=-4 u_{X} U-8 r_{X} \mathrm{e}^{2 r} R-8 r_{X}^{3} \mathrm{e}^{2 r} .
$$

This together with the embedding of $H^{1}(\mathbb{R})$ in the space of continuous functions allows us to write the estimate

$$
\left\|H_{r}(T)\right\|_{L^{2}}+\left\|H_{u}(T)\right\|_{L^{2}} \leq Q\left(\|(r(T), u(T))\|_{H^{2}}\right)
$$


for some monotone increasing function $Q$. Using now identity (4.7) and the key estimate (4.15), we arrive at

$$
\frac{d}{d T}\left(E_{\operatorname{lin}}\left(r_{X X}(T), u_{X X}(T)\right)+E(r, u)\right) \leq Q_{1}\left(\|(r(T), u(T))\|_{H^{2}}\right)
$$

with $E_{\operatorname{lin}}\left(r_{X X}, u_{X X}\right):=\left(e^{2 r}, u_{X X}^{2}\right)_{L^{2}}+2\left(e^{4 r}, r_{X X}^{2}\right)_{L^{2}}$. Moreover, using (4.11) together with its analogue for $E_{\text {lin }}$ and assumption (4.14), we get

$$
\begin{aligned}
C^{-1} e^{-4\left\|r_{0}\right\|_{L^{\infty}}}\|u(T), r(T)\|_{H^{2}}^{2} \leq E_{\operatorname{lin}}\left(r_{X X}(T), u_{X X}(T)\right)+ & E(r(T), u(T)) \leq \\
& \leq C e^{4\left\|r_{0}\right\|_{L^{\infty}}}\|u(T), r(T)\|_{H^{2}}^{2}
\end{aligned}
$$

for all $T \leq T_{0}$ if the extra assumption (4.13) is satisfied. Integrating now (4.16) in time $T \leq T_{0}$ and using the last estimate, we arrive at

$$
\sup _{T \leq T_{0}} \|\left(r(T), u(T) \|_{H^{2}}^{2} \leq Q_{2}\left(\left\|\left(r_{0}, u_{0}\right)\right\|_{H^{2}}\right)+T_{0} Q_{3}\left(\sup _{T \leq T_{0}}\|(r(T), u(T))\|_{H^{2}}\right)\right.
$$

for some monotone increasing functions $Q_{2}$ and $Q_{3}$. This estimate allows us to fix the time interval $T_{0}=T_{0}\left(\left\|\left(r_{0}, u_{0}\right)\right\|_{H^{2}}\right)$ to be small enough in order to get the desired estimate

$$
\|(r(T), u(T))\|_{H^{2}} \leq 2 Q_{2}\left(\left\|\left(r_{0}, u_{0}\right)\right\|_{H^{2}}\right), \quad \forall T \leq T_{0},
$$

which coincides with (4.10) for $s=2$. Finally, shrinking further the lifespan $T_{0}$ of the solution if necessary, we satisfy assumption (4.13) as well. This finishes the derivation of (4.10) for $s=2$. Existence and uniqueness of the solution is straightforward when the proper a priori estimate is verified. Thus, the theorem is proved.

\section{Approximate solutions of the perturbed WMEs}

In this section we study the finite-order Taylor expansion in $\varepsilon$ of $(\widehat{A}, \widehat{\phi})$ in (1.3) . Substitution of (1.3) into (1.1) gives the exact equations

$$
\begin{aligned}
\partial_{T} \widetilde{\phi}+\left(k+\partial_{X} \widetilde{\phi}\right)^{2}-\gamma \widetilde{A}^{2}+\omega-\varepsilon^{2} \widetilde{A}^{-1} \partial_{X}^{2} \widetilde{A} & =0 \\
\partial_{T} \widetilde{A}+2\left(k+\partial_{X} \widetilde{\phi}\right) \partial_{X} \widetilde{A}+A \partial_{X}^{2} \widetilde{\phi} & =0
\end{aligned}
$$

As before, we assume that $\gamma=\omega+k^{2}$ (the general case is reduced to this particular one by scaling). These equations are expressed in terms of $\widetilde{\phi}$ as they will be needed below for estimates on the phase. However, the estimates on the Taylor expansions will be carried out in $(\widetilde{r}, \widetilde{u})$ variables. We transform the equations in two steps. First introduce the new variable $\widetilde{r}$ defined by $\widetilde{A}=e^{\widetilde{r}}$ to eliminate the singularity at $\widetilde{A}=0$. Then (15.1) reads

$$
\begin{aligned}
\partial_{T} \widetilde{\phi}+\left(k+\partial_{X} \widetilde{\phi}\right)^{2}-\gamma e^{2 \widetilde{r}}+\gamma-k^{2}-\varepsilon^{2} \partial_{X}^{2} \widetilde{r}-\varepsilon^{2}\left(\partial_{X} \widetilde{r}\right)^{2} & =0 \\
\partial_{T} \widetilde{r}+2\left(k+\partial_{X} \widetilde{\phi}\right) \partial_{X} \widetilde{r}+\partial_{X}^{2} \widetilde{\phi} & =0 .
\end{aligned}
$$


Differentiating the first equation in $X$ and inserting $\widetilde{u}=\partial_{X} \widetilde{\phi}$, we end up with

$$
\begin{aligned}
\partial_{T} \widetilde{u}+\partial_{X}(k+\widetilde{u})^{2}-\gamma \partial_{X} e^{2 \widetilde{r}}-\varepsilon^{2} \partial_{X}^{3} \widetilde{r}-\varepsilon^{2} \partial_{X}\left(\partial_{X} \widetilde{r}\right)^{2} & =0 \\
\partial_{T} \widetilde{r}+2(k+\widetilde{u}) \partial_{X} \widetilde{r}+\partial_{X} \widetilde{u} & =0
\end{aligned}
$$

which is a perturbed version of SWEs (4.1) studied earlier. These equations (5.1)-(5.3) are exact. However, at this stage we will prove the existence of $\varepsilon^{2 n}$-approximations $(\widehat{r}, \widehat{\phi})$ to the exact solution $(\widetilde{r}, \widetilde{\phi})$ only.

We construct the approximate solution $(\widehat{r}, \widehat{u})$ for equations (5.3) and then lift the result to the initial equations (5.1) to obtain phase information. The approximate solution $(\widehat{r}, \widehat{u})$ will be constructed in the class of square integrable functions (in agreement with Theorem 4.2). However, the functions $\widehat{A}$ and $\widehat{\phi}$ are not square integrable in general, but will satisfy the following integrability properties:

$$
\widehat{A}-1, \partial_{T} \widehat{A}, \partial_{X} \widehat{A}, \partial_{T} \widehat{\phi}, \partial_{X} \widehat{\phi} \in H^{s}(\mathbb{R})
$$

for some $s>0$.

Assume that the smooth local solution $(r(T), u(T))$ of the limit system (4.1) is given and satisfies (4.10) for some $s \geq 2$. The existence of such a solution is confirmed in Theorem 4.2 for some small $T_{0}$ depending on the initial data, but in this section the lifespan of this given solution is not assumed to be small.

Expand the approximate solution $(\widehat{r}, \widehat{u})$ into a Taylor series in $\varepsilon$ :

$$
\begin{aligned}
\widehat{r}(T, \varepsilon) & :=r(T)+\varepsilon^{2} r_{1}(T)+\varepsilon^{4} r_{2}(T)+\cdots+\varepsilon^{2 n} r_{n}(T), \\
\widehat{u}(T, \varepsilon) & :=u(T)+\varepsilon^{2} u_{1}(T)+\varepsilon^{4} u_{2}(T)+\cdots+\varepsilon^{2 n} u_{n}(T) .
\end{aligned}
$$

Then, inserting these expansions into (5.3) and equating terms with equal powers of $\varepsilon$, we get the recursive equations

$$
\left\{\begin{array}{l}
\partial_{T} r_{l}=-\partial_{X} u_{l}-2(u+k) \partial_{X} r_{l}-2 \partial_{X} r u_{l}+H_{l-1, r}(T),\left.\quad r_{l}\right|_{T=0}=0 \\
\partial_{T} u_{l}=-2 \partial_{X}\left((u+k) u_{l}\right)-2 \partial_{X}\left(e^{2 r} r_{l}\right)+H_{l-1, u}(T),\left.\quad u_{l}\right|_{T=0}=0
\end{array}\right.
$$

where $l \geq 1$ and $\left(r_{0}, u_{0}\right)=(r, u)$. The smooth functions $H_{l-1, r}$ and $H_{l-1, u}$ depend only on $r, r_{1}, \cdots r_{l-1}$ and $u, u_{1}, \cdots, u_{l-1}$ and their derivatives up to order 3 , and so the sequence of linear equations (5.6) can be solved recursively. Moreover, the residual $\widehat{\operatorname{Res}}_{n}(T)$ satisfies

$$
\widehat{\operatorname{Res}}_{n, \star}=\varepsilon^{2(n+1)} R_{n, \star}\left(\varepsilon, D u, D u_{1}, \cdots, D u_{n}, D r, D r_{1}, \cdots, D r_{n}\right)
$$

for some smooth functions $R_{n, \star}$. Here " $\star$ " represents " $r$ " or " $u$ " and $D u$ means the collection of all $X$-derivatives of $u$ up to order 3 . For instance,

$$
H_{0, r}=0, \quad H_{0, u}=\partial_{X}^{3} r+\partial_{X}\left(\partial_{X} r\right)^{2}
$$

and 


$$
\begin{aligned}
R_{1, r}=2 u_{1} \partial_{X} r_{1}, \quad R_{1, u}=2 u_{1} \partial_{X} u_{1}-\gamma \varepsilon^{-4} \partial_{X} & \left(e^{2 r}\left(e^{2 \varepsilon^{2} r_{1}}-1-2 \varepsilon^{2} r_{1}\right)\right)- \\
& -\partial_{X}^{3} r_{1}-2 \partial_{X}\left(\partial_{X} r \partial_{X} r_{1}\right)-\varepsilon^{2} \partial_{X}\left(\partial_{X} r_{1}\right)^{2} .
\end{aligned}
$$

Thus, in order to get the desired result for the approximate solutions $(\widehat{r}, \widehat{u})$, we just need to get good estimates for the $H^{s}$-norms of the solutions for the linear problems (5.6). This is possible due to Proposition 4.1 and gives the following result.

Proposition 5.1. Let $s \geq 0$ and $n \in \mathbb{N}$ be fixed and let $(r(T), u(T)), t \leq T_{0}$ be a solution of the limit problem (4.1) satisfying

$$
\|r(t)\|_{H^{s+3 n+5}(\mathbb{R})}+\|u(T)\|_{H^{s+3 n+5}(\mathbb{R})} \leq C .
$$

Then there exists an approximate solution $(\widehat{r}, \widehat{u})$ which satisfies

$$
\left\{\begin{array}{l}
\partial_{T} \widehat{r}=-\partial_{X} \widehat{u}-2(\widehat{u}+k) \partial_{X} \widehat{r}+\widehat{\operatorname{Res}}_{n, r}(T), \\
\partial_{T} \widehat{u}=\varepsilon^{2} \partial_{X}^{3} \widehat{r}-\partial_{X}((\widehat{u}+k))^{2}+\varepsilon^{2} \partial_{X}\left(\partial_{X} \widehat{r}\right)^{2}+\gamma \partial_{X}\left(e^{2 \widehat{r}}\right)+\widehat{\operatorname{Res}}_{n, u}(T),
\end{array}\right.
$$

defined on the same time interval $T \in\left[0, T_{0}\right]$ such that

$$
\|\widehat{r}(T)\|_{H^{s+5}}+\|\widehat{u}(T)\|_{H^{s+5}}+\left\|\partial_{T} \widehat{r}(T)\right\|_{H^{s+2}} \leq C_{1},
$$

and

$$
\left\|\widehat{\operatorname{Res}}_{n, r}(T)\right\|_{H^{s+2}}+\left\|\widehat{\operatorname{Res}}_{n, u}(T)\right\|_{H^{s+2}} \leq C_{1} \varepsilon^{2(n+1)},
$$

for all $T \in\left[0, T_{0}\right]$, and for some constant $C_{1}$ which is independent of $\varepsilon$.

\subsection{Lifting to the phase equation}

Let us now lift the approximate solution $(\widehat{r}, \widehat{u})$ to the phase variables in equation (5.1). The principal difficulty here is defining the approximate phase $\widehat{\phi}$ (going from (5.3) to (5.2)), as the lifting from equations (5.2) to (5.1) is immediate.

For exact solutions we have the relation $u=\partial_{X} \phi$, so the obvious way to define the approximate phase would be to integrate over the slow space variable:

$$
\widehat{\phi}(T, X)=\int_{0}^{X} \widehat{u}(T, s) d s+\bar{\phi}(T) ;
$$

this strategy is used in [8]. However, in this case it is not clear how to determine the function $\bar{\phi}(T)$, and so a complete picture is lacking. We propose an alternative way, namely, to use the first equation of (5.2) to solve for the phase,

$$
\partial_{T} \widehat{\phi}:=-(k+\widehat{u})^{2}+\gamma e^{2 \widehat{r}}-\gamma+k^{2}+\varepsilon^{2} \partial_{X}^{2} \widehat{r}+\varepsilon^{2}\left(\partial_{X} \widehat{r}\right)^{2},\left.\quad \widehat{\phi}\right|_{T=0}=\phi_{0} .
$$

Then, since $(\widehat{r}, \widehat{u})$ is already defined, the phase $\widehat{\phi}(T)$ will be restored in a unique way. From this formula we see that indeed $\partial_{T} \widehat{\phi}(T) \in H^{s+3}(\mathbb{R})$ for $T \leq T_{0}$. Moreover, comparing this definition with the second equation of (5.10), we see that

$$
\partial_{T}\left(\partial_{X} \widehat{\phi}-\widehat{u}\right)=-\widehat{\operatorname{Res}}_{n, u}(T),\left.\quad \partial_{X} \widehat{\phi}\right|_{T=0}-\left.\widehat{u}\right|_{T=0}=0
$$


and therefore

$$
\partial_{X} \widehat{\phi}(T)=\widehat{u}(T)-\int_{0}^{T} \widehat{\operatorname{Res}}_{n, u}(s) d s .
$$

Inserting this identity into the right-hand side of equation (5.13) and to the first equation of (5.10), we conclude that the pair $(\widehat{r}, \widehat{\phi})$ solves

$$
\begin{aligned}
\partial_{T} \widehat{\phi}+\left(k+\partial_{X} \widehat{\phi}\right)^{2}-\gamma e^{2 \widehat{r}}+\gamma-k^{2}-\varepsilon^{2} \partial_{X}^{2} \widehat{r}-\varepsilon^{2}\left(\partial_{X} \widehat{r}\right)^{2} & =\operatorname{Res}_{n, \phi}(T) \\
\partial_{T} \widehat{r}+2\left(k+\partial_{X} \widehat{\phi}\right) \partial_{X} \widehat{r}+\partial_{X}^{2} \widehat{\phi} & =\operatorname{Res}_{n, r}(T),
\end{aligned}
$$

where

$$
\operatorname{Res}_{n, \phi}(T)=-\left(2 k+2 \widehat{u}(T)-\int_{0}^{T} \widehat{\operatorname{Res}}_{n, u}(s) d s\right) \int_{0}^{T} \widehat{\operatorname{Res}}_{n, u}(s) d s
$$

and

$$
\operatorname{Res}_{n, r}(T)=\widehat{\operatorname{Res}}_{n, r}(T)-\int_{0}^{T} \partial_{X} \widehat{\operatorname{Res}}_{n, u}(s) d s-2 \partial_{X} \widehat{r} \int_{0}^{T} \widehat{\operatorname{Res}}_{n, u}(s) d s .
$$

Thus, we have proved the following result which can be considered as the main result of this section.

Theorem 5.2. Let the assumptions of Proposition 5.1 hold and suppose $(\widehat{r}, \widehat{u})$ is the approximate solution constructed there. Then the function $\widehat{A}:=e^{\widehat{r}}$ and $\widehat{\phi}$ defined by (5.13) satisfy

$$
\begin{aligned}
\partial_{T} \widehat{\phi}+\left(k+\partial_{X} \widehat{\phi}\right)^{2}-\gamma \widehat{A}^{2}+\gamma-k^{2}-\varepsilon^{2} \widehat{A}^{-1} \partial_{X}^{2} \widehat{A} & =\operatorname{Res}_{n, \phi} \\
\partial_{T} \widehat{A}+2\left(k+\partial_{X} \widehat{\phi}\right) \partial_{X} \widehat{A}+A \partial_{X}^{2} \widehat{\phi} & =\operatorname{Res}_{n, A},
\end{aligned}
$$

where the residuals $\operatorname{Res}_{n, \phi}$ and $\operatorname{Res}_{n, r}$ are defined by (5.15) and (5.16) respectively, the residual $\operatorname{Res}_{n, A}:=e^{\widehat{r}} \operatorname{Res}_{n, r}$ and the following estimate holds:

$$
\left\|\operatorname{Res}_{n, A}(T)\right\|_{H^{s+1}}+\left\|\operatorname{Res}_{n, \phi}(T)\right\|_{H^{s+1}} \leq C \varepsilon^{2(n+1)},
$$

for $T \leq T_{0}$. The approximate solution $(\widehat{A}, \widehat{\phi})=\left(e^{\widehat{r}}, \widehat{\phi}\right)$ also satisfies the estimate

$$
\|\widehat{r}(T)\|_{H^{s+5}}+\left\|\partial_{X} \widehat{\phi}(T)\right\|_{H^{s+2}}+\left\|\partial_{T} \widehat{r}(T)\right\|_{H^{s+2}} \leq C
$$

for $T \leq T_{0}$ and some constant $C$ which is independent of $\varepsilon$.

Remark 5.3. Restoring the phase $\widehat{\phi}$ via equation (5.13) gives more detailed information about the phase then one might expect. First of all, with this approach, the phase is restored in a unique way which, in turn, leads to the construction of an approximate solution of the initial NLS also in a unique way and this is crucial for our proof of validity, see next section below.

In addition, it follows from equation (5.13) that $\partial_{T} \widehat{\phi} \in L^{2}$ and, therefore, $\widehat{\phi}(T)-\widehat{\phi}(0) \in L^{2}$ which gives a useful extra information about the approximate phase.

Finally, our approach allows to verify that the difference $\widetilde{\phi}-\widehat{\phi}$ between the exact and approximate phases is in $L^{2}$ and is controlled by the appropriate power of $\varepsilon$ (although the details of such estimates are outside the scope of this paper). 


\section{Exact solutions in Sobolev spaces}

In this section the properties of the exact solution (1.7) needed to complete the proof of Theorem 1.1 are established. It is assumed that the two estimates (5.18) and (5.19) hold with $s=0$.

Express the exact solution in the form

$$
\widetilde{\Psi}(T, X, \varepsilon)=\widehat{\Psi}(T, X, \varepsilon) V(T, X, \varepsilon), \quad V=1+W_{1}+\mathrm{i} W_{2},
$$

where $\widehat{\Psi}$ is the approximation of (1.3) to $n$-th order obtained in $\$ 5$ and $W_{1}+\mathrm{i} W_{2}$ is an unknown complex-valued function. Substitution of this expression into the cubic NLS equation (1.1) gives the following equation:

$$
\mathrm{i} \partial_{T} V+\varepsilon \partial_{X}^{2} V+2 \varepsilon \frac{\partial_{X} \widehat{\Psi}}{\widehat{\Psi}} \partial_{X} V+\frac{\mathrm{i} \partial_{T} \widehat{\Psi}+\varepsilon \partial_{X}^{2} \widehat{\Psi}}{\widehat{\Psi}} V+\gamma \varepsilon^{-1}|\widehat{\Psi}|^{2}|V|^{2} V=0
$$

Recall that, according to (1.3),

$$
\widehat{\Psi}(T, X, \varepsilon)=\mathrm{e}^{\widehat{r}} \mathrm{e}^{\mathrm{i} \varepsilon^{-1} \widehat{\Theta}}, \widehat{\Theta}:=\omega T+k X+\widehat{\phi}
$$

where $W_{1}, W_{2}, \widehat{\Theta}, \widehat{r}$, and $\widehat{\phi}$ are all functions of $(T, X, \varepsilon)$, we obtain that

$$
\frac{\mathrm{i} \partial_{T} \widehat{\Psi}+\varepsilon \partial_{X}^{2} \widehat{\Psi}}{\widehat{\Psi}} V=i \widehat{A}^{-1}\left(\partial_{T} \widehat{A}+\widehat{A} \partial_{X}^{2} \widehat{\Theta}+2 \partial_{X} \widehat{A} \partial_{X} \widehat{\Theta}\right)-\varepsilon^{-1} \mathrm{i}\left(\partial_{T} \widehat{\Theta}+\left(\partial_{X} \widehat{\Theta}\right)^{2}-\widehat{A}^{-1} \partial_{X}^{2} \widehat{A}\right)
$$

Using now that the functions $\widehat{A}$ and $\widehat{\phi}$ satisfy equation (5.17) together with the dispersion relation $\gamma=\omega+k^{2}$, we arrive at the following equation for $V$ :

$$
\begin{aligned}
\mathrm{i} \partial_{T} V+\varepsilon \partial_{X}^{2} V & +2 \mathrm{i}\left(k+\partial_{X} \widehat{\phi}\right) \partial_{X} V+2 \varepsilon \partial_{X} \widehat{r} \partial_{X} V+ \\
& +\gamma e^{2 \widehat{r}} \varepsilon^{-1} V\left(|V|^{2}-1\right)+\left(\mathrm{i} e^{-\widehat{r}} \operatorname{Res}_{A}-\varepsilon^{-1} \operatorname{Res}_{\phi}\right) V=0 .
\end{aligned}
$$

Separating real and imaginary parts, (6.2) splits into an equation for $W_{1}$ and $W_{2}$,

$$
\left\{\begin{aligned}
\partial_{T} W_{1}=-\varepsilon\left(\partial_{X}^{2}+2 \partial_{X} \widehat{r} \partial_{X}\right) W_{2}-2\left(k+\partial_{X} \widehat{\phi}\right) \partial_{X} W_{1}+ \\
+ \\
\operatorname{Re}\{\mathcal{H}(T)(1+W)\}+\varepsilon^{-1} F_{1}(W) \\
\partial_{T} W_{2}=\varepsilon\left(\partial_{X}^{2}+2 \partial_{X} \widehat{r} \partial_{X}+2 \varepsilon^{-2} \gamma e^{2 \widehat{r}}\right) W_{1}-2\left(k+\partial_{X} \widehat{\phi}\right) \partial_{X} W_{2}+ \\
+\operatorname{Im}\{\mathcal{H}(T)(1+W)\}+\varepsilon^{-1} F_{2}(W)
\end{aligned}\right.
$$

where the non-linearity $F$ is a polynomial containing only quadratic and cubic terms and

$$
\mathcal{H}:=e^{-\widehat{r}} \operatorname{Res}_{A}+\mathrm{i} \varepsilon^{-1} \operatorname{Res}_{\phi} .
$$

Thus, in these new coordinates the functions $W_{1}$ and $W_{2}$ are responsible for the deviation

of the exact solution $\widetilde{\Psi}(T, X)$ from the approximate solution $\widehat{\Psi}(T, X)$ constructed via the higher order WMT, so our task here is to verify that these functions are in a sense small. 
In order to solve the semilinear equation (6.3) on the interval $T \leq T_{0}$ it is enough to get good estimates in the $H^{s}$-norm for the linearized non-homogeneous equation:

$$
\left\{\begin{array}{l}
\partial_{T} W_{1}=-\varepsilon\left(\partial_{X}^{2}+2 \partial_{X} \widehat{r} \partial_{X}\right) W_{2}-2\left(k+\partial_{X} \widehat{\phi}\right) \partial_{X} W_{1}+H_{1}(T) \\
\partial_{T} W_{2}=\varepsilon\left(\partial_{X}^{2}+2 \partial_{X} \widehat{r} \partial_{X}+2 \varepsilon^{-2} \gamma e^{2 \widehat{r}}\right) W_{1}-2\left(k+\partial_{X} \widehat{\phi}\right) \partial_{X} W_{2}+H_{2}(T)
\end{array}\right.
$$

where $H_{i}(T)$ are given external forces. This is done in the following theorem.

Theorem 6.1. Let the above conditions on $\widehat{r}$ and $\widehat{\phi}$ hold and let $\gamma=-1$. Then, for every $W(0) \in H^{1}(\mathbb{R})$, there exists a unique solution $W \in C\left(\left[0, T_{0}\right], H^{1}\right)$ for any $T_{0} \in \mathbb{R}_{+}$of $(6.5)$ and this solution satisfies the following estimate:

$$
\begin{aligned}
\left\|\partial_{X} W(T)\right\|_{L^{2}}^{2}+\varepsilon^{-2} & \left\|W_{1}(T)\right\|_{L^{2}}^{2}+\left\|W_{2}(T)\right\|_{L^{2}}^{2} \leq \\
\leq & C\left(\left\|\partial_{X} W(0)\right\|_{L^{2}}^{2}+\varepsilon^{-2}\left\|W_{1}(0)\right\|_{L^{2}}^{2}+\left\|W_{2}(0)\right\|_{L^{2}}^{2}\right) e^{C T}+ \\
& \quad+C \int_{0}^{T} e^{C(T-\tau)}\left(\left\|\partial_{X} H(\tau)\right\|_{L^{2}}^{2}+\varepsilon^{-2}\left\|H_{1}(\tau)\right\|_{L^{2}}^{2}+\left\|H_{2}(\tau)\right\|_{L^{2}}^{2}\right) d \tau,
\end{aligned}
$$

where the constant $C$ is independent of $\varepsilon$.

Proof. We restrict attention to a formal verification of the key estimate (6.6). The existence of a solution can then be confirmed a posteriori in a standard way using an appropriate approximation scheme.

Fix $\gamma=-1$, multiply the first and the second equations in (6.5) by $-\partial_{X}\left(e^{2 \widehat{r}} \partial_{X} W_{1}\right)+$ $2 \varepsilon^{-2} e^{4 \widehat{r}} W_{1}$ and $-\partial_{X}\left(e^{2 \widehat{r}} \partial_{X} W_{2}\right)$, integrate over $X$, take a sum, and use the following obvious identity:

$$
\partial_{X}^{2} W+2 \widehat{r}_{X} \partial_{X} W=e^{-2 \widehat{r}} \partial_{X}\left(e^{2 \widehat{r}} \partial_{X} W\right)
$$

After cancellation of the leading terms in the right-hand side and integration by parts, this gives

$$
\begin{array}{r}
\frac{1}{2} \frac{d}{d T}\left(\left\|e^{\widehat{r}} \partial_{X} W\right\|_{L^{2}}^{2}+2 \varepsilon^{-2}\left\|e^{2 \widehat{r}} W_{1}\right\|_{L^{2}}^{2}\right)-\left(\partial_{T} \widehat{r},\left(e^{\widehat{r}} \partial_{X} W\right)^{2}\right)- \\
-4 \varepsilon^{-2}\left(\partial_{T} \widehat{r},\left(e^{2 \widehat{r}} W_{1}\right)^{2}\right)=-\left(e^{2 \widehat{r}} \partial_{X}\left(e^{-2 \widehat{r}}\left(k+\partial_{X} \widehat{\phi}\right)\right),\left|e^{\widehat{r}} \partial_{X} W\right|^{2}\right)+ \\
+2 \varepsilon^{-2}\left(e^{-4 \widehat{r}}\left(e^{4 \widehat{r}}\left(k+\widehat{\phi}_{X}\right)\right)_{X},\left|e^{2 \widehat{r}} W_{1}\right|^{2}\right)+\left(H_{1}(T),-\partial_{X}\left(e^{2 \widehat{r}} \partial_{X} W_{1}\right)+2 \varepsilon^{-2} e^{4 \widehat{r}} W_{1}\right)+ \\
+\left(H_{2}(T),-\partial_{X}\left(e^{2 \widehat{r}} \partial_{X} W_{2}\right)\right) .
\end{array}
$$

Using (5.19) with $s=0$, we have

$$
\left\|\partial_{T} \widehat{r}\right\|_{L^{\infty}}+\|\widehat{r}\|_{W^{1, \infty}}+\left\|\partial_{X} \widehat{\phi}\right\|_{W^{1, \infty}} \leq C,
$$

and therefore (6.7) can be transformed to 


$$
\begin{aligned}
& \frac{d}{d T}\left(\left\|e^{\widehat{r}} \partial_{X} W\right\|_{L^{2}}^{2}+2 \varepsilon^{-2}\left\|e^{2 \widehat{r}} W_{1}\right\|_{L^{2}}^{2}\right)- \\
& \quad-C\left(\left\|e^{\widehat{r}} \partial_{X} W\right\|_{L^{2}}^{2}+2 \varepsilon^{-2}\left\|e^{2 \widehat{r}} W_{1}\right\|_{L^{2}}^{2}\right) \leq C\left(\left\|\partial_{X} H(T)\right\|_{L^{2}}^{2}+\varepsilon^{-2}\left\|H_{1}(T)\right\|_{L^{2}}^{2}\right) .
\end{aligned}
$$

The Gronwall inequality then gives the following estimate:

$$
\begin{aligned}
\left\|\partial_{X} W\right\|_{L^{2}}^{2}+2 \varepsilon^{-2}\left\|W_{1}\right\|_{L^{2}}^{2} \leq C\left(\left\|\partial_{X} W(0)\right\|_{L^{2}}^{2}+\varepsilon^{-2}\left\|W_{1}(0)\right\|_{L^{2}}^{2}\right) e^{C T}+ & \\
& +C \int_{0}^{T} e^{C(T-\tau)}\left(\left\|\partial_{X} H(\tau)\right\|_{L^{2}}^{2}+\varepsilon^{-2}\left\|H_{1}(\tau)\right\|_{L^{2}}^{2}\right) d \tau
\end{aligned}
$$

which coincides with (6.6) up to the $L^{2}$-norm of $W_{2}$ which we now need to estimate. To this end, multiply the second equation of (6.5) by $W_{2}$ and integrate over $X$. This gives

$$
\begin{aligned}
\frac{1}{2} \frac{d}{d t}\left\|W_{2}\right\|_{L^{2}}^{2}=-\varepsilon\left(\partial_{X} W_{1}, \partial_{X} W_{2}\right) & +2 \varepsilon\left(\partial_{X} \widehat{r} \partial_{X} W_{1}, W_{2}\right)+ \\
+ & 2 \gamma \varepsilon^{-1} e^{2 \widehat{r}}\left(W_{1}, W_{2}\right)-2\left(\left(k+\partial_{X} \widehat{\phi}\right) \partial_{X} W_{2}, W_{2}\right)+\left(H_{2}, W_{2}\right) .
\end{aligned}
$$

Using again (5.19) together with Cauchy-Schwarz inequality, we get

$$
\frac{1}{2} \frac{d}{d T}\left\|W_{2}\right\|_{L^{2}}^{2}-C\left\|W_{2}\right\|_{L^{2}}^{2} \leq C\left(\varepsilon\left\|\partial_{X} W\right\|_{L^{2}}^{2}+\varepsilon^{-2}\left\|W_{1}\right\|_{L^{2}}^{2}+\left\|H_{2}(T)\right\|_{L^{2}}^{2}\right),
$$

which together with (6.9) gives

$$
\begin{aligned}
& \left\|W_{2}(T)\right\|_{L^{2}}^{2} \leq C\left(\left\|\partial_{X} W(0)\right\|_{L^{2}}^{2}+\varepsilon^{-2}\left\|W_{1}(0)\right\|_{L^{2}}^{2}+\left\|W_{2}(0)\right\|_{L^{2}}^{2}\right) e^{C T}+ \\
& \quad+C \int_{0}^{T} e^{C(T-s)}\left(\left\|\partial_{X} H(s)\right\|_{L^{2}}^{2}+\varepsilon^{-2}\left\|H_{1}(s)\right\|_{L^{2}}^{2}+\left\|H_{2}(s)\right\|_{L^{2}}^{2}\right) d s,
\end{aligned}
$$

which gives the desired estimate (6.6) and finishes the proof of the theorem.

Remark 6.2. We estimate the solution $W$ of problem (6.5) in the space $H^{1}$ only, which is sufficient for the present purposes. The analogue of this estimate in $H^{s}$ with $s>1$ can be verified analogously to the proof of Proposition 4.1 by differentiating equation (6.5) in $X$ sufficiently many times.

We are now ready to return to the non-linear equation (6.3) with zero initial data. For this we need $\mathcal{H}$ in (6.4) to satisfy

$$
\mathcal{H}(T) \sim O\left(\varepsilon^{2 n+1}\right),
$$

and this follows from the estimate (5.18) with $s=0$. Now, inverting the linear part and using (6.6) together with the fact that $H^{1}$ is an algebra we arrive at

$$
\|W(T)\|_{H^{1}}^{2} \leq C \varepsilon^{-2} \sup _{T \leq T_{0}}\left(\|\mathcal{H}(T)(1+W(T))\|_{H^{1}}^{2}+\varepsilon^{-2}\|F(W(T))\|_{H^{1}}^{2}\right) \leq
$$




$$
\begin{aligned}
\leq C \varepsilon^{4 n} & +C \varepsilon^{4 n} \sup _{T \leq T_{0}}\|W(T)\|_{H^{1}}^{2}+ \\
& +C \varepsilon^{-4} \sup _{T \leq T_{0}}\|W(T)\|_{H^{1}}^{4}\left(1+\sup _{T \leq T_{0}}\|W(T)\|_{H^{1}}^{2}\right) .
\end{aligned}
$$

Let $\widetilde{W}:=\varepsilon^{-2 n} W$. Then, the new function satisfies

$$
\|\widetilde{W}(T)\|_{H^{1}}^{2} \leq C+C \varepsilon^{4 n} \sup _{T \leq T_{0}}\|\widetilde{W}(T)\|_{H^{1}}^{2}+C \varepsilon^{8 n-4} \sup _{T \leq T_{0}}\|\widetilde{W}(T)\|_{H^{1}}^{4}\left(1+\varepsilon^{4 n} \sup _{T \leq T_{0}}\|\widetilde{W}(T)\|_{H^{1}}^{2}\right)
$$

and we see that in the case $n \geq 1$, the obtained estimate guarantees that $\|\widetilde{W}(T)\|_{H^{1}} \leq 2 C$ for all $T \in\left[0, T_{0}\right]$ for some $T_{0}>0$ which is independent of $\varepsilon \rightarrow 0$. Thus, we have proved the following result.

Corollary 6.3. Let the above assumptions hold and suppose $n \geq 1$. Then, for $\varepsilon$ small enough, equation (6.3) with zero initial data possesses a unique solution $W(T)$ on the time interval $T \leq T_{0}$, and the following estimate holds:

$$
\sup _{T \leq T_{0}}\|W(T)\|_{H^{1}(\mathbb{R})} \leq C \varepsilon^{2 n} .
$$

The constant $C$ depends on the norms of approximating solution, but is independent of $\varepsilon$.

Estimate (6.12) is a standard corollary of (6.11) and the existence of a solution can be obtained using the semigroup technique since (6.3) has a semilinear structure.

This corollary gives the required estimate for the distance between exact and approximate solutions of the NLS, recalling that, by definition,

$$
W(T)=(\widetilde{\Psi}(T)-\widehat{\Psi}(T)) e^{-\mathrm{i} \varepsilon^{-1} \widehat{\Theta}(T)},
$$

thereby completing the proof of Theorem 1.1 .

\section{Linear stability of wavetrains and validity}

A surprise in Theorem 1.1 is that the power of $\varepsilon$ in the estimate (1.9) is $\varepsilon^{2 n}$ rather than the more natural $\varepsilon^{2 n+1}$. In this section we show why $\varepsilon^{2 n}$ is essential and can not be improved. It is intimately connected with an algebraic instability of the wavetrain (1.2).

Firstly, in contrast to the Gevrey spaces approach developed in [8], the spectral stability of the background wavetrain is a necessary condition for the validity of Whitham approximations in Sobolev spaces. For this reason, it looks natural to use the energy norms related with this spectral stability in the proof of validity. Actually, our presentation of the exact solution in the form of (6.1) and the choice of the energy norm for equation (6.5) are inspired by the spectral stability arguments.

The linearization of the initial NLS (1.1) on a wavetrain solution $e^{\mathrm{i} k x+\mathrm{i} \omega t}$ reads

$$
\mathrm{i} \partial_{t} Z+\partial_{x}^{2} Z+\gamma\left(2 Z+e^{2(\mathrm{i} k x+\mathrm{i} \omega t)} \bar{Z}\right)=0, \quad \gamma=\omega+k^{2} .
$$


In order to make this equation autonomous, let $Z=e^{\mathrm{i} k x+\mathrm{i} \omega t} W$, giving

$$
\partial_{t} W-\mathrm{i} \partial_{x}^{2} W+2 k \partial_{x} W-\mathrm{i} \gamma(W+\bar{W})=0
$$

or, separating, real and imaginary parts,

$$
\left\{\begin{array}{l}
\partial_{t} W_{1}=-\partial_{x}^{2} W_{2}-2 k \partial_{x} W_{1} \\
\partial_{t} W_{2}=\partial_{x}^{2} W_{1}-2 k \partial_{x} W_{2}+2 \gamma W_{1}
\end{array}\right.
$$

which coincides with the unscaled version $(\varepsilon=1)$ of equation (6.5) with $\widehat{r}=\widehat{\phi}=0$ and zero right-hand sides.

This equation possesses the conservation law

$$
\frac{d}{d t}\left(\left\|\partial_{x} W\right\|_{L^{2}}^{2}-2 \gamma\|\operatorname{Re} W\|_{L^{2}}^{2}\right)=0
$$

which gives the spectral stability of the wavetrain for $\gamma<0$ and also is a prototype for our key estimate (6.6).

Note also that (7.3) gives the stability of the linearized equation in the space

$$
\left(W_{1}, W_{2}\right) \in H^{1} \times \dot{H}^{1},
$$

where $\dot{H}^{1}$ is the corresponding homogeneous Sobolev space, which is enough for spectral stability, but does not imply the stability in a natural phase space $H^{1} \times H^{1}$ or $L^{2} \times L^{2}$.

Actually, a bit more accurate analysis shows that problem (7.1) is unstable in a natural space $H^{1} \times H^{1}$, namely, the $L^{2}$-norm of $W_{2}(t)$ may grow (at most linearly in time) despite the fact that $\left\|\partial_{x} W_{2}(t)\right\|$ remains bounded. As usual, such an instability is caused by a Jordan cell. Indeed, after the Fourier transform in $x$, (7.1) reads

$$
\frac{d}{d t}\left(\begin{array}{l}
\mathcal{F} W_{1} \\
\mathcal{F} W_{2}
\end{array}\right)=\mathbb{M}(\xi)\left(\begin{array}{l}
\mathcal{F} W_{1} \\
\mathcal{F} W_{2}
\end{array}\right), \quad \mathbb{M}(\xi):=\left(\begin{array}{cc}
-2 k \mathrm{i} \xi, & \xi^{2} \\
-\xi^{2}-\gamma, & -2 k \mathrm{i} \xi
\end{array}\right)
$$

and we see the Jordan cell in $\mathbb{M}(\xi)$ at $\xi=0$ which is responsible for the long-wave instability and produces the linear growth of the $L^{2}$-norm of $W_{2}(t)$ in time.

In the scaled variables this linear growth produces an extra factor $\varepsilon^{-1} T \leq \varepsilon^{-1} T_{0}$ in the corresponding estimates. In particular, the factor $\varepsilon^{-2}$ in the middle part of (6.11) is caused exactly by this instability. In turn, this decreases the power of $\varepsilon$ in the right-hand side of Theorem 1.1 from the expected $\varepsilon^{2 n+1}$ to $\varepsilon^{2 n}$ and requires the use of the higher order Whitham approximations in $\$ 5$.

\section{Concluding remarks}

The main result of the paper is validity of the Whitham theory as an approximation to solutions that modulate a periodic travelling wave of the NLS equation. However, the theory does not rely on the structure of NLS and so will carry over to other equations, particularly nonintegrable nonlinear wave equations, when the Whitham equations are strictly hyperbolic, 
and the appropriate energy functionals can be constructed. As shown in \$7, replacing $\varepsilon^{2 n}$ with $\varepsilon^{2(n+1)}$ on the right-hand side of (1.9) in Theorem 1.1 is unavoidable if we are working in $H^{1}$. However, there is still a room for refining the estimates utilizing homogeneous Sobolev spaces where this instability disappears.

An extension of interest is validity of multiphase WMT as an approximation to modulation of multiphase periodic travelling waves of the coupled nonlinear Schrödinger equation. A proof of validity in Gevrey spaces for this case has been given in [3]. The problem with multiphase WMEs is that the characteristics can be elliptic, hyperbolic, or mixed [4]. Gevrey spaces are indifferent to characteristic type, but the only hope for a proof of validity in Sobolev spaces for the multiphase WMEs for coupled NLS is to restrict to the case where all characteristics are hyperbolic, and do not change type with time.

\section{Acknowledgements}

The research of AK and SZ is partially supported by EPSRC Grant EP/P024920/1 on Finitedimensional reduction, inertial manifolds, and homoclinic structures in dissipative PDEs and the work of SZ is also supported by the grant 19-71-30004 of Russian Science Foundation.

\section{References}

[1] S. Benzoni-Gavage, P. Noble, \& L.M. Rodrigues. Slow modulations of periodic waves in Hamiltonian PDEs, with application to capillary fluids, J. Nonl. Sci. 24 711-768 (2014).

[2] T.J. Bridges. Symmetry, Phase Modulation, and Nonlinear Waves, Cambridge University Press: Cambridge (2017).

[3] T.J. Bridges, A. Kostianko, \& G. Schneider. A proof of validity for multiphase Whitham modulation theory, Proc. Roy. Soc. Lond. A 4762020203 (2020).

[4] T.J. BRIDGes \& D.J. RATlifF. Krein signature and Whitham modulation theory: the sign of characteristics and the "sign characteristic", Stud. Appl. Math. 142 314-355 (2019).

[5] J.C. Bronski, V. Hur, \& M.A. Johnson. Modulational instability in equations of KdV type, in New Approaches to Nonlinear Waves, Edited by E. ToBiscH, Lect. Notes. Phys. 908 83-133 (2016).

[6] J.C. Bronski \& M.A. Johnson. The modulational instability for a generalized Korteweg-de Vries equation, Arch. Rat. Mech. Anal. 197 357-400 (2010).

[7] R. CARles. Semi-Classical Analysis for Nonlinear Schrödinger Equations, World Scientific: Singapore (2008).

[8] W.-P. Düll \& G. SCHNEIDER. Validity of Whitham's equations for the modulation of periodic traveling waves in the NLS equation, J. Nonl. Sci. 19 453-466 (2009).

[9] P. GÉRARD. Remarques sur l'analyse semi-classique de l'équation de Schrödinger non linéaire, Séminaire EDP de l'Ecole Polytechnique, Palaiseau, France (1992-93), lecture no. XIII. 
[10] B. Grebert \& T. KAPPELER. The defocusing NLS equation and its normal form, European Mathematical Society, Lecture Notes Series 18, DOI 10.4171/131 (2014).

[11] E. Grenier. Semiclassical limit of the nonlinear Schrödinger equation in small time, Proc. Amer. Math. Soc. 126 523-530 (1998).

[12] S. Jin, C.D. Levermore, \& D.W. McLaughlin. The semiclassical limit of the defocusing NLS hierarchy, Comm. Pure Appl. Math. 52 613-654 (1999).

[13] A.M. Kamchatnov. Nonlinear Periodic Waves and Their Modulations, World Scientific: Singapore (2000).

[14] A. MAJdA. Compressible fluid flow and systems of conservation laws in several space variables, Appl. Math. Sci 53, Springer: Berlin (1984).

[15] G. Schneider \& H. UECKer. Nonlinear PDEs: A Dynamical Systems Approach, American Mathematical Society: Providence (2017).

[16] G.B. Whitham. Linear and Nonlinear Waves, Wiley-Interscience: New York (1974). 